\title{
Multiple food-borne trematodiases with profound systemic involvement: a case report and literature review
}

\author{
Lun Li ${ }^{1}$, Xinchao Liu², Baotong Zhou², Shimin Zhang ${ }^{3}$, Geng Wang ${ }^{3}$, Guotao Ma ${ }^{4}$, Ruxuan Chen ${ }^{1}$, Yang Zou ${ }^{5}$, \\ Wei CaO ${ }^{2^{*}}$ and Taisheng $\mathrm{Li}^{2}$
}

\begin{abstract}
Background: Food-borne trematodiases are an important group of neglected global diseases. Affected patients in regions with low prevalence usually experience delayed diagnosis, especially when presenting with atypical clinical symptoms. Here, we presented a rare case of a Chinese patient infected with three food-borne trematodiases.

Case presentation: A 42-year-old man presented with diarrhea, lower extremity edema, and symptoms of cardiac dysfunction. He had a history of intermittent consumption of raw freshwater fishes for 6-7 years. Upon evaluation, he had eosinophilia, anemia, intrahepatic bile duct dilatation and a growing space-occupying lesion in the left atrium. The patient underwent a cardiac surgery which revealed an endocardial hematoma due to mechanical injuries. Imaging investigations also revealed intracranial and pulmonary lesions. A total of three trematodiases were diagnosed based upon microscopic stool examination, from which eggs of Clonorchis sinensis, Heterophyidae and Echinostomatidae were identified. Deposition of Clonorchis sinensis eggs was also observed from ileocecal squash slides. The patient was successfully treated with three cycles of praziquantel.
\end{abstract}

Conclusions: Food-borne trematodiases may present with systemic involvement. Patients with dietary history of high risk or atypical ingestions should be evaluated for parasitic infection, even in non-endemic areas.

Keywords: Trematodiases, Food-borne, Ectopic lesion

\section{Background}

Foodborne parasitic diseases remain largely underestimated worldwide, with the highest prevalence reported in East and Southeast Asia [1]. Food-borne trematodes are classified into liver, intestinal, and lung flukes, based on their primary sites of infection. However, a few flukes may migrate substantially within the body, leading to systematic damage involving the skin, abdomen, heart, or central nervous system (CNS) [1-3]. Uncommon clinical manifestations may potentially delay diagnosis and treatment in non-endemic areas. Although immunodiagnostic techniques and molecular methods can present important clues, microscopic examination is always the definitive means of diagnosis. Here we report a

\footnotetext{
* Correspondence: wcao_pumch@163.com

${ }^{2}$ Department of Infectious Diseases, Peking Union Medical College Hospital, Chinese Academy of Medical Sciences, \#1 Shuaifuyuan, Dongcheng District, Beijing 100730, China

Full list of author information is available at the end of the article
}

case of a critically ill patient infected with multiple trematodes, complicated by ectopic parasitism.

\section{Case presentation}

A 42-year-old man presented to the emergency room with intermittent diarrhea for over four months and progressive lower extremity edema for three months. He had 5-6 bowel movements per day, with no obvious fever or abdominal discomfort. Progressive pitting edema of the bilateral legs was noticed one month later, accompanied by a decline in exertional tolerance. The patient lost approximately $15 \mathrm{~kg}$ over this period of time. A series of echocardiographic examinations had revealed a growing mass in his left atrium of uncertain origin, which grew from $25 \times 22$ to $60 \times 54 \mathrm{~mm}$ within 3 months. His previous medical history was remarkable for poorly controlled type 2 diabetes mellitus complicated by diabetic nephropathy, retinopathy and 
peripheral neuropathy. He also had a five-year history of major depressive disorder without regular treatment. $\mathrm{He}$ lived with his mother and sister in the city of Qingdao, Shandong Province, and denied any recent travel history. The patient's sister reported that he had intermittently consumed raw river fish for as long as 6-7 years before onset of this episode. Due to the mental status of the patient, his medical history was also obtained and confirmed by his mother and sister who lived with him.

On admission, the patient appeared emaciated and anemic. He was afebrile, his blood pressure was 93/67 $\mathrm{mmHg}$, and heart rate $102 \mathrm{bpm}$. He was moody, disoriented, and slightly hypoxemic with an oxygen saturation of $90 \%$ at room air. A grade II diastolic rumbling murmur was heard at the apex. Examination of his lungs and abdomen was otherwise unremarkable. Decreased myodynamia of the bilateral limbs was appreciated, more significant on the left side, with a positive left Babinski sign.

Initial laboratory assessment revealed peripheral eosinophilia (eosinophils $6.64 \times 10^{9} / \mathrm{L}$ ) and anemia (hemoglobin $85 \mathrm{~g} / \mathrm{L}$ ). The level of gamma-glutamyl transpeptidase was elevated at $1093 \mathrm{U} / \mathrm{L}$, and alkaline phosphatase was $666 \mathrm{U} / \mathrm{L}$, with no apparent hyperbilirubinemia (Table 1). Serial of electrocardiograms showed paroxysmal atrial fibrillation. A bedside echocardiogram was immediately arranged, and a space-occupying lesion measuring $60 \times 54 \mathrm{~mm}$ was identified in the left atrium with a slight pericardial effusion (Fig. 1a). The left ventricular ejection fraction (LVEF) was moderately reduced at the level of $55 \%$. The initial chest CT showed scattered bilateral pulmonary infiltrations and pleural effusion, with a cavity in the right upper lobe. Abdominal

Table 1 Laboratory data for the case patient

\begin{tabular}{lll}
\hline Test & $\begin{array}{l}\text { Case } \\
\text { patient }\end{array}$ & $\begin{array}{l}\text { Reference Range, } \\
\text { Adults }\end{array}$ \\
\hline Leukocytes, $\times 10^{9} / \mathrm{L}$ & 14.57 & $3.5-9.5$ \\
Eosnophils, $\times 10^{9} / \mathrm{L}$ & 6.65 & $0.02-0.50$ \\
Eosnophils, \% & 45.6 & $0.5-5$ \\
Erythrocyte, $\times 10^{12} / \mathrm{L}$ & 3.1 & $4-5.5$ \\
Hemoglobin, g/L & 85 & $120-160$ \\
Platelets, $\times 10^{9} / \mathrm{L}$ & 325 & $100-350$ \\
Albumin, g/L & 37 & $35-52$ \\
Total bilirubin, $\mu$ mol/L & 5.6 & $5.1-22.2$ \\
Aspartate aminotransferase, IU/L & 22 & $15-40$ \\
Alanine aminotransferase, IU/L & 12 & $9-50$ \\
Gamma-glutamyl transpeptidase, & 1473 & $10-60$ \\
IU/L & & \\
Alkaline phosphatase, IU/L & 905 & $45-125$ \\
Urea, mmol/L & 9.65 & $2.78-7.14$ \\
Creatinine, $\mu$ mol/L & 97 & $59-104$ \\
\hline
\end{tabular}

CT scan showed intrahepatic bile duct dilatation with no obvious obstruction (Fig. 1b). A CT scan of the head was also preformed due to recurrent episodes of seizures during hospitalization, and showed low density lesions in bilateral corona radiate.

The initial differential diagnosis included infectious endocarditis, Löffler endocarditis, hypereosinophilic syndrome, parasitic infection and hypersensitive reactions to recent medication. Although no evidence of fungus was identified from qualified sputum samples, sulperazon and caspofungin were initiated empirically based on the CT manifestation and history of uncontrolled diabetes. Supportive care was also initiated. A multidisciplinary discussion was held, and consensus was achieved regarding the urgency of cardiac surgery, both to alleviate progressive heart failure and to obtain a tissue specimen for diagnostic purposes. The patient underwent the surgery two weeks following admission. Surprisingly, the cardiac mass turned out to be an endocardial hematoma, possibly due to mechanical perforation of the left ventricular posterior wall close to the base of posterior mitral valve (Fig. 1c). The valves were otherwise intact. Pathological review of the specimen revealed nonspecific inflammation and all pathogenic examinations turned out to be unremarkable.

The surgical findings largely ruled out endocarditis of infectious or autoimmune origin, nor did they reveal any evidence of eosinophilic infiltration. Given the systemic involvement and the dietary habits mentioned by his family, we began to consider the possibility of parasitic infection with systemic involvement. A more detailed history confirmed raw or half-raw consumption of freshwater fish and shrimps, frogs, tadpoles and snake gall bladders nearly every month in recent years as a result of his altered mental status. Further tests were warranted. Multiple stool samples were sent for microscopic examination by direct fecal smear, and various eggs were identified. Eggs of Clonorchis sinensis were first identified (measuring $27-30 \mu \mathrm{m} \times 16-18 \mu \mathrm{m}$, Fig. 1d), at a count of 3-5/low-power (LP) field. Clonorchiasis was therefore confirmed, which partially explained the patient's chronic diarrhea and dilatation of intrahepatic bile ducts. However, this liver fluke rarely causes such widespread organ damage. A few days later, another type of egg smaller than that of $C$. sinensis was identified, which was morphologically most consistent with that of heterophyid trematodes (measuring 23-26 $\mu \mathrm{m} \times 13-15 \mu \mathrm{m}$, Fig. 1d). The eggs of Heterophyidae were much fewer in number, and required 10-20 LP field to find one. Moreover, several large-sized eggs were also observed (measuring $100-110 \mu \mathrm{m} \times 60-70 \mu \mathrm{m}$ ), consistent with those of the Echinostomatidae family (Fig. 1e). Approximately five eggs of the Echinostomatidae could be identified per fecal smear. Further evaluations included a contrast- 


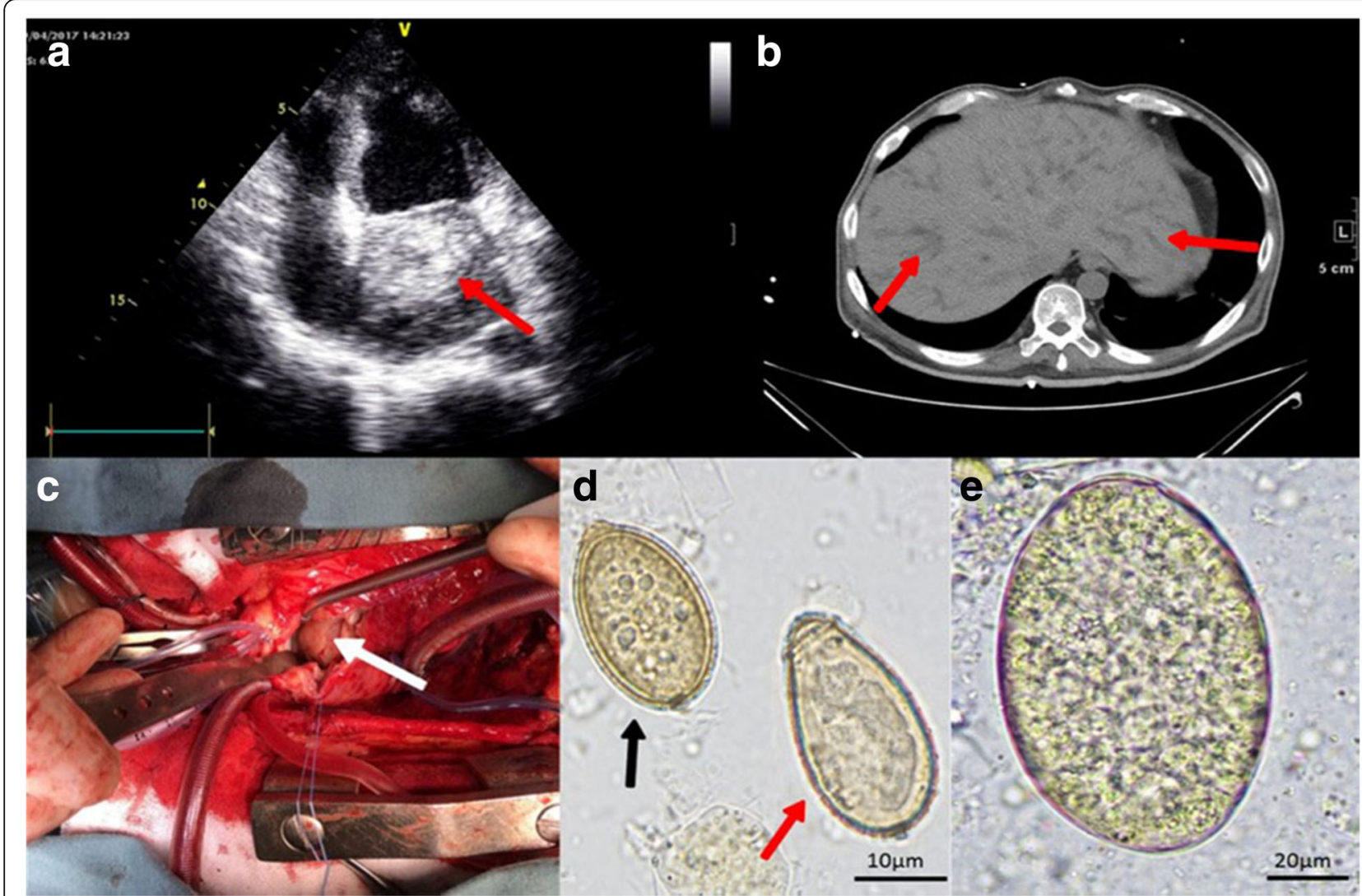

Fig. 1 Imaging, surgical and pathogenic findings in the patient. (a) A space-occupying lesion (red arrow) was observed in the left atrium measured $60 \times 54 \mathrm{~mm}$ by cardiac ultrasonography. (b) Abdominal CT scan showed extensive intrahepatic bile duct dilatation (red arrow). (c) The round cardiac mass (white arrow) in the left atrium was exposed in the surgery, which was an endocardial hematoma close to the posterior mitral valve. (d) The egg of C. sinensis (red arrow) was firstly identified in the stool samples, and later a smaller egg (black arrow) consistent with that of heterophyid trematodes was distinguished from C. sinensis. (e) Larger-sized eggs in the stool samples were consistent with those of the Echinostomatidae family

enhanced cerebral magnetic resonance imaging test with angiography, which revealed multiple long T2 densities in the centrum semiovale with no obvious vascular involvement. Lumbar puncture was performed and showed moderately elevated intracranial pressure at $210 \mathrm{mmH}_{2} \mathrm{O}$ and a slightly elevated protein level in the cerebral spinal fluid. A colonoscopy was apparently normal, but deposition of C. sinensis eggs was later observed on ileocecal squash slides. Specific PCR band patterns of C. sinensis were later observed in samples of the ileocecal tissues and stool, but not in the sputum or heart tissues. DNA of heterophyid trematodes was not detected from any obtained samples. The primers of PCR were designed according to previous studies (Additional file 1: Table S1).

Once the diagnosis of triple trematodiases was established, treatment with praziquantel was started. Due to the comprehensive involvement and the concern for hypersensitivity reaction, a reduced oral dose of praziquantel at $25 \mathrm{mg} / \mathrm{kg} / \mathrm{d}$ was initiated for the first 10 days combined with low-dose dexamethasone. The patient tolerated this regimen well and received the full dose of
$75 \mathrm{mg} / \mathrm{kg} / \mathrm{d}$ for ten days each month for another consecutive two months. His diarrhea gradually resolved with a steady improvement in nutritional status and cardiac function. Continuous surveillance of his stool samples revealed no further trematode eggs since the second month of treatment. Follow-up CT scans showed remission of pulmonary and liver lesions, while enhanced MRI showed absorption of previous abnormal signals in centrum semiovale.

\section{Discussion and conclusions}

Clonorchiasis is a widespread foodborne infectious disease and has been reported in 24 provinces in China. However, the prevalence varies, and infections of families Echinostomatidae and Heterophyidae are rarely reported especially in the northern areas [4]. Infections are most frequently acquired through eating uncooked freshwater fish or other aquatic products containing encysted organisms. The present patient represented a very rare case of multiple trematode co-infections including $C$. sinensis, Heterophyidae and Echinostomatidae, confirmed by parasitological 
stool examination. His clinical manifestations provided an indication of the sites, load and intensity of parasitic infection, as well as the host immune status.

Mild to moderate infection of any of the three trematodes may present with unspecific symptoms, while severe forms may lead to symptoms such as fatigue, weight loss, anemia, abdominal pain and diarrhea $[5,6]$. The triple trematodes infection, especially the heavy burden of C. sinensis in this case, is sufficient to explain the gastrointestinal manifestations, emaciation and anemia of this patient. Characteristic cholestasis with intrahepatic bile duct dilatation caused by clonorchiasis was also observed in this patient. However, involvement of the heart, brain and lungs are not commonly seen in trematodes infection, yet we did not find any evidence of autoimmune diseases, malignancies or other infections which could explain such comprehensive damage. As far as we know, among the three kinds of trematodes we identified, Heterophyidae is the only one whose eggs can enter the blood circulation and cause ectopic parasitism, especially in immunocompromised hosts. Until now, ectopic infection has not been described in clonorchiasis or echinostomiasis. Based on this knowledge and the patient's response to treatment, we deduced that it was most probable that heterophyid ectopic parasitism led to lesions of the brain, lung and heart in this case (Table 2). The most frequently reported sites of infection for heterophyidiasis are the heart valves and the brain, which could be potentially fatal $[1-3,7-9]$. However, it was reasonable to hypothesize that attachment of heterophyid eggs to the surface of the endocardium could cause necrosis and subsequent perforation, resulting in the endocardial hematoma.

The most straightforward and classic way for diagnosing parasitic infection is the detection of eggs or the parasite itself via morphological examination, as we did in this case. The detection of eggs in the stool is the standard for diagnosis of trematodiases. Detailed identification by means of delicate microscopic examination revealed Metagonimus yokogawai as the most likely species. However, further specification of Echinostomatidae was not possible through visual speculation. Immunodiagnostic techniques and molecular methods have been developed as an important supplement to diagnosis. However, false negative results are possible due to the testing methods, disease severity and pathogenesis of the infection. Moreover, cases with a low burden of eggs and/or unsuccessful cracking of eggs during DNA extraction can also lead to amplification failure on molecular testing, which might explain the difficulty achieving greater specificity in terms of each organism in this case $[10,11]$. Nevertheless, ectopic infiltration and subsequent necrosis of surrounding tissue remained the most probable explanation for the mechanical injuries leading to this patient's heart failure.

Praziquantel is the drug of choice for several species of trematodes including clonorchiasis from liver fluke infection, as well as heterophyiasis and echinostomiasis from intestinal fluke infection. The recommended dosage of $25 \mathrm{mg} / \mathrm{kg}$ three times daily for $2-3$ days can be highly efficacious for clonorchiasis [1], but patients with a heavy pathogen load may require multiple courses [12]. Moreover, a single oral dose of $10-20 \mathrm{mg} / \mathrm{kg}$ praziquantel is reported to be sufficient for a treatment of heterophyiasis and echinostomiasis, but no prior reports have involved such diffuse and multiple fluke infection. Therefore, we adopted a progressive strategy with three cycles of therapy (starting with a low-dose praziquantel cycle combined with dexamethasone), to reduce allergic reaction and recurrence.

The patient described here represents a very rare case of multi-fluke infection with systemic involvement. Parasitic infection is one of the essential differential diagnoses of eosinophilia, but atypical manifestations may lead to delays or neglect in considering the diagnosis. Physicians in non-endemic areas need to raise their awareness of and keep in mind the signs of fluke infection. In particular, parasitic infection should be considered in cases with significant eosinophilia or relevant risk factors in the patient's history. Immunodiagnostic techniques and molecular methods are newer alternatives for the detection of parasites in fecal samples, but microscopic examination remains the definitive diagnostic tool.

Table 2 Trematodes infection and related manifestation for the case patient

\begin{tabular}{lllll}
\hline Parasitic sites & Symptom/Manifestation & Trematode species & Diagnostic evidence & Possibility \\
\hline Biliary system & Intrahepatic bile duct dilatation and liver damage & C. sinensis & Eggs identified and PCR positive in stool & Proven \\
Gastrointestinal tract & Diarrhea and weight loss & C. sinensis & Eggs identified and PCR positive in stool; Proven & Eggs deposited in ileocecal \\
& & Heterophyidae & Eggs identified in stool & Proven \\
& & Echinostomatidae & Eggs identified in stool & Proven \\
Heart & Endocardial hematoma and heart failure & Heterophyidae & Mechanic perforation of the ventricular wall Probably \\
Brain & Seizures and decreased myodynamia & Heterophyidae & & Possibly \\
Lung & Infiltrations and cavity & Heterophyidae & & Possibly \\
\hline
\end{tabular}




\section{Additional file}

Additional file 1: Table S1. Primers for amplification by polymerase chain reaction (PCR). (DOCX $19 \mathrm{~kb})$

\section{Abbreviations}

CNS: Central nervous system; CSF: Cerebral spinal fluid; LVEF: Left ventricular ejection fraction; PCR: Polymerase chain reaction

\section{Acknowledgements}

Not applicable.

\section{Authors' contributions}

WC (corresponding author), $\mathrm{XL}$ and $\mathrm{LL}$ were in charge of management and treatment of the patient. $\mathrm{LL}$ and RC collected and interpreted the clinical data, and drafted the manuscript. BZ interpreted the clinical data. WC and TL revised the manuscript critically for important content. GM performed heart operation and provided details of surgery. SZ and GW carried out the microbiological examination. YZ interpreted the clinical data and performed nucleotide sequencing. All authors read and approved the final manuscript.

\section{Funding}

This report received no specific grant from any funding agency in the public, commercial, or not-for-profit sectors.

\section{Availability of data and materials}

Essential data and materials were presented in this manuscript. More clinical, laboratory, and imaging data are available from the corresponding author on reasonable request.

\section{Ethics approval and consent to participate}

We obtained ethics approval from the Peking Union Medical College

Hospital review boards.

\section{Consent for publication}

Written informed consent was obtained from the patient and his sister for publication of this case report and the images in it. A copy of the written consent is available by request.

\section{Competing interests}

The authors declare that they have no competing interests.

\section{Author details}

'Department of Internal Medicine, Peking Union Medical College Hospital, Chinese Academy of Medical Sciences, Beijing 100730, China. ${ }^{2}$ Department of Infectious Diseases, Peking Union Medical College Hospital, Chinese Academy of Medical Sciences, \#1 Shuaifuyuan, Dongcheng District, Beijing 100730, China. ${ }^{3}$ Department of Clinical Laboratory, Peking Union Medical College Hospital, Chinese Academy of Medical Sciences, Beijing 100730, China. ${ }^{4}$ Department of Cardiac Surgery, Peking Union Medical College Hospital, Chinese Academy of Medical Sciences, Beijing 100730, China. ${ }^{5}$ Beijing Institute of Tropical Medicine, Beijing Friendship Hospital, Capital Medical University, Beijing Key Laboratory for Research on Prevention and Treatment of Tropical Diseases, Beijing 100050, China.

Received: 20 January 2019 Accepted: 29 May 2019

Published online: 14 June 2019

\section{References}

1. Control of foodborne trematode infections. Report of a WHO study group. World Health Organ Tech Rep Ser. 1995;849:1-157.

2. Sithithaworn P, Sripa B, Kaewkes S, Haswell-Elkins M. Food-borne trematodes. In: Cook GC, Zumla Al, editors. Manson's tropical diseases. London: W. B. Saunders; 2009. p. 1461-76.

3. Chai JY. Intestinal flukes. In: Murrell KD, Fried B, editors. World class parasites Vol. 11. Dordrecht. Netherlands: Springer; 2007. p. 53-115.

4. Li X. Food-borne parasitic zoonoses in the People's Republic of China. Southeast Asian J Trop Med Public Health. 1991;22(Suppl):31-5.

5. Chai JY, Lee SH. Food-borne intestinal trematode infections in the Republic of Korea. Parasitol Int. 2002;51(2):129-54.
6. Graczyk TK, Fried B. Echinostomiasis: a common but forgotten food-borne disease. Am J Trop Med Hyg. 1998:58(4):501-4.

7. Africa CM, Leon WD, Garcia EY. Visceral complications in intestina heterophyidiasis of man. Acta Med Philipp. 1940:447-9.

8. Belizario VY Jr, Bersabe MJ, de Leon WU, Hilomen VY, Paller GV, de Guzman AD Jr, Bugayon MG. Intestinal heterophyidiasis: an emerging food-borne parasitic zoonosis in southern Philippines. Southeast Asian J Trop Med Public Health. 2001;32(Suppl 2):36.

9. Chai JY, Seo BS, Lee SH. Studies on intestinal trematodes in Korea XI. Two cases of human infection by Heterophyes heterophyes nocens. Kisaengchunghak Chapchi the Korean Journal of Parasitology. 1984:22(1):37.

10. Sripa B, Traub RJ, Macaranas J, Mungthin M, Leelayoova S, Cribb T, Murrell $\mathrm{KD}$, Thompson RCA. A new PCR-based approach indicates the range of Clonorchis sinensis now extends to Central Thailand. PLoS Negl Trop Dis. 2009;3(1):e367.

11. Stensvold CR, Saijuntha W, Sithithaworn P, Wongratanacheewin S, Strandgaard H, Ornbjerg N, Johansen MV. Evaluation of PCR based coprodiagnosis of human opisthorchiasis. Acta Trop. 2006;97(1):26-30.

12. Tinga N, De N, Vien HV, Chau L, Toan ND, Kager PA, Vries PJ. Little effect of praziquantel or artemisinin on clonorchiasis in northern Vietnam. A pilot study. Tropical Med Int Health. 1999;4(12):814-8.

\section{Publisher's Note}

Springer Nature remains neutral with regard to jurisdictional claims in published maps and institutional affiliations.
Ready to submit your research? Choose BMC and benefit from:

- fast, convenient online submission

- thorough peer review by experienced researchers in your field

- rapid publication on acceptance

- support for research data, including large and complex data types

- gold Open Access which fosters wider collaboration and increased citations - maximum visibility for your research: over $100 \mathrm{M}$ website views per year

At BMC, research is always in progress.

Learn more biomedcentral.com/submissions 